\title{
Erratum to: How buyers forecast: Buyer-seller relationship as a boundary condition of the impact bias
}

\author{
Ashwani Monga • Haipeng (Allan) Chen • \\ Michael Tsiros • Mona Sinha
}

Published online: 13 April 2011

(C) Springer Science+Business Media, LLC 2011

\section{Erratum to: Mark Lett \\ DOI 10.1007/s11002-010-9133-y}

Author Mona Srivastava has reverted back to her maiden name.

The author name should now read as Mona Sinha.

The online version of the original article can be found at http://dx.doi.org/10.1007/s11002-010-9133-y.

\footnotetext{
A. Monga $(\bowtie)$

Marketing Department, Moore School of Business, University of South Carolina, 1705 College Street,

Columbia, SC 29212, USA

e-mail: ashwani@moore.sc.edu

H. (Allan) Chen

Marketing Department, Mays Business School, Texas A\&M University,

College Station,

TX 77843, USA

e-mail: hchen@mays.tamu.edu

M. Tsiros

Marketing Department, University of Miami, 501 Kosar/Epstein, Coral Gables, FL 33124, USA

e-mail: tsiros@miami.edu

\section{Tsiros}

Tassos Papastratos Research Professor of Marketing, ALBA Graduate Business School, Athens, Greece
}

M. Sinha

Harvard Business School India Research Center, Mahindra Towers, Worli, Mumbai, India e-mail: monas@hbs.edu 